\title{
Critical Currents: Just How Critical Are They?
}

\author{
David C Larbalestier
}

\begin{abstract}
Large scale superconductivity is trying to do something novel and risky, that is to break out of present important markets served by the low temperature superconductors (LTS) $\mathrm{Nb}-\mathrm{Ti}$ and $\mathrm{Nb}_{3} \mathrm{Sn}$, where superconductivity has little competition, to new markets served by high temperature superconductors (HTS) where superconductivity competes head to head with copper and iron. The controlling technical parameter for this breakout is high current density $\left(J_{c}\right)$, when achieved in a useful conductor form at favorable cost. The development of attractive HTS conductors has much to learn from the experience acquired in developing LTS conductors, especially as $\mathrm{Nb}_{3} \mathrm{Sn}$ is now undergoing significant new attempts to push its performance/cost envelope. Nb47-49 wt.\% Ti is the work horse conductor for more than $90 \%$ of all superconducting magnets. It is a very well developed material for which it is well understood how to make the material homogeneous on all scales except the nanoscale, where it can be made heterogeneous on scales of $1-5 \mathrm{~nm}$ so as to provide very strong flux pinning without paying any fabrication penalty. $\mathrm{Nb}_{3} \mathrm{Sn}$ is longitudinally homogeneous but radially quite inhomogeneous, because the diffusion process needed to form the brittle phase cannot be allowed to go to completion without growing the $\sim 100 \mathrm{~nm}$ grain size that is responsible for strong flux pinning. By contrast, flux pinning in HTS conductors is poorly understood because they are heterogeneous on many length scales, causing current to percolate inhomogeneously through the filaments past many barriers, of which high angle grain boundaries are an important example. The present generation of HTS conductors are made from approximately uniaxially aligned BSCCO-2223. They are demonstrating that significant prototype motors, power cables, fault current limiters etc. can be made, but conductor costs are currently too high, partly because the current density is compromised by their high percolation inefficiency. Better in-field current densities are available with biaxially-aligned YBCO, which has higher percolation efficiency and stronger flux pinning. This paper reviews the present state of high-Jc conductor development, focusing on the complex interplay between physics, fabrication and materials issues. A full version of the paper was not available at conference publication time, but it will appear in a future issue of IEEE Transactions on Applied Superconductivity. An accessible version of the talk is available at http://www.asc.wisc.edu/.
\end{abstract}

Index Terms-Niobium titanium, niobium tin, Bi-2223, Y-123.

Manuscript received September 19, 2000. This work was supported in part by AFOSR, DOE and NSF through the MRSEC program.

D. C. Larbalestier is with the Applied Superconductivity Center, the Department of Materials Science and Engineering, and the Department of Physics, University of Wisconsin, Madison WI 53706 USA (telephone: 608263-2194, e-mail: larbalestier@engr.wisc.edu). 